University of New Hampshire

University of New Hampshire Scholars' Repository

Space Science Center

Institute for the Study of Earth, Oceans, and

Space (EOS)

2001

\title{
Energetic proton spectra in the 11 June 1991 solar flare
}

\author{
C A. Young \\ Goddard Space Flight Center \\ K Bennett \\ ESTEC \\ A Connors \\ Eureka Scientific \\ R Diehl \\ Max-Planck-Institut für extraterrestriche \\ Mark L. McConnell \\ University of New Hampshire - Main Campus, mark.mcconnell@unh.edu
}

See next page for additional authors

Follow this and additional works at: https://scholars.unh.edu/ssc

Part of the Astrophysics and Astronomy Commons

\section{Recommended Citation}

Energetic proton spectra in the 11 June 1991 solar flare Young, C. A. and Bennett, K. and Connors, A. and Diehl, R. and McConnell, M. and Rank, G. and Ryan, J. M. and Suleiman, R. and Schönfelder, V. and Winkler, C., AIP Conference Proceedings, 587, 623-627 (2001), DOI:http://dx.doi.org/10.1063/1.1419474

This Conference Proceeding is brought to you for free and open access by the Institute for the Study of Earth, Oceans, and Space (EOS) at University of New Hampshire Scholars' Repository. It has been accepted for inclusion in Space Science Center by an authorized administrator of University of New Hampshire Scholars' Repository. For more information, please contact Scholarly.Communication@unh.edu. 


\section{Authors}

C A. Young, K Bennett, A Connors, R Diehl, Mark L. McConnell, G Rank, James M. Ryan, R Suleiman, V Schonfelder, and C Winkler 


\section{AIP $\mid$ proceedings}

\section{Energetic proton spectra in the 11 June 1991 solar flare}

C. A. Young, K. Bennett, A. Connors, R. Diehl, M. McConnell, G. Rank, J. M. Ryan, R. Suleiman, V. Schönfelder , and C. Winkler

Citation: AIP Conference Proceedings 587, 623 (2001); doi: 10.1063/1.1419474

View online: http://dx.doi.org/10.1063/1.1419474

View Table of Contents: http://scitation.aip.org/content/aip/proceeding/aipcp/587?ver=pdfcov

Published by the AIP Publishing

\section{Articles you may be interested in}

Propagation of impulsive solar energetic particle events

AIP Conf. Proc. 598, 353 (2001); 10.1063/1.1434024

The mixing of interplanetary magnetic field lines: A significant transport effect in studies of the energy spectra of impulsive flares

AIP Conf. Proc. 528, 47 (2000); 10.1063/1.1324280

Energetic proton spectra in the 11 June 1991 solar flare

AIP Conf. Proc. 510, 564 (2000); 10.1063/1.1303266

Accelerated-particle spectral variability in the 1991 June 11 solar flare

AIP Conf. Proc. 510, 559 (2000); 10.1063/1.1303265

Energetic particles in the solar wind and at the Sun

AIP Conf. Proc. 385, 25 (1997); 10.1063/1.51744 


\title{
Energetic Proton Spectra in the 11 June 1991 Solar Flare
}

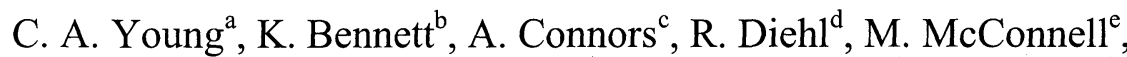 \\ G. Rank ${ }^{\mathrm{d}}$, J. M. Ryan ${ }^{\mathrm{e}}$, R. Suleiman ${ }^{\mathrm{f}}$, V. Schönfelder ${ }^{\mathrm{d}}$, C. Winkler ${ }^{\mathrm{b}}$ \\ ${ }^{a}$ Emergent-IT, Inc., Goddard Space Flight Center \\ ${ }^{b}$ European Space Research and Technology Center \\ ${ }^{c}$ Eureka Scientific \\ ${ }^{d}$ Max Planck Institute for Extraterrestrial Physics \\ ${ }^{e}$ University of New Hampshire \\ ${ }^{f}$ Havard-Smithsonian Center for Astrophysics
}

\begin{abstract}
The June 11,1991 gamma-ray flare seen by the Compton Gamma-ray Observatory (CGRO) displays several features that make it a dynamic and rich event. It is a member of a class of long duration gamma-ray events with both $2.223 \mathrm{MeV}$ and greater than $8 \mathrm{MeV}$ emission for hours after the impulsive phase. It also contains an inter-phase between the impulsive and extended phases that presents a challenge to the standard gamma-ray line (GRL) flare picture. This phase has strong $2.223 \mathrm{MeV}$ emission and relatively weak $4.44 \mathrm{MeV}$ emission indicative of a very hard parent proton spectrum. However, this would indicate emission greater than $8 \mathrm{MeV}$, which is absent from this period. We present the application of new spectroscopy techniques to this phase of the flare in order to present a reasonable explanation for this seemly inconsistent picture.
\end{abstract}

During the $22^{\text {nd }}$ solar cycle NOAA active region 6659 crossed the solar disk from 1 June to 15 June 1991 and produced some of the largest flares of that cycle. Six X-class flares occurred on 1,4,6,9,11, and 15 June 1991. After the X12 flare on 4 June 1991, the Sun was declared a CGRO target-of-opportunity and CGRO was re-oriented toward the Sun on 9 June 1991. This placed the Sun into the FoV of all CGRO's instruments from 9 to 15 June 1991 . On 11 June 1991 a X12/3B flare started at 0156 UT as measured by the 1-8 $\AA$ SXR channel of GOES-7. The flare sit was at a heliographic location of N31W17. COMPTEL measured $\gamma$-ray emission from 0.8 to 30 $\mathrm{MeV}$ and neutrons for several hours (Ryan et al. 1993; McConnell et al. 1994; Suleiman et al. 1994). This included nuclear line emission, $2.223 \mathrm{MeV}$ emission lasting over 5 hours (Rank 1996) and 8-30 MeV $\pi^{0}$ decay emission. The EGRET spark chamber could not observe the impulsive phase due to dead-time effects but observed $>1 \mathrm{GeV}$ emission for at least 8 hours after the peak. The EGRET spectrum showed no sign of a high-energy cut-off (Kanbach et al. 1993). EGRET/TASC impulsive phase measurements of $2.223 \mathrm{MeV}$ emission and nuclear line emission were reported (Dunphy et al. 1999; Schneid et al. 1994) along with evidence for $\pi^{0}$ emission, neutrons and spectral evolution (Dunphy et al. 1999). OSSE reported prolonged 2.223 $\mathrm{MeV}$ emission (Murphy et al. 1993) and nuclear emission, $0.511 \mathrm{MeV}$ positronannihilation emission, $>16 \mathrm{MeV} \gamma$-rays and neutrons. BATSE-LADs measured HXRs and $\gamma$ rays for about one hour in the energy range of $20 \mathrm{keV}$ to $\sim 1.9 \mathrm{MeV}$. CGRO was not the only $\gamma$-ray experiment that observed the 11 June 1991 flare. GRANAT/PHEBUS also reported observations of Bremsstrahlung, $2.223 \mathrm{MeV}$, and nuclear emission during the impulsive phase of the flare (Trottet et al. 1993;1994).

CP587, GAMMA 2001: Gamma-Ray Astrophysics 2001, edited by S. Ritz et al. (C) 2001 American Institute of Physics 0-7354-0027-X/01/\$18.00 
Figure 1 shows a lightcurve of the 11 June 1991 phase with both the Telescope and Burst modes of COMPTEL. The flare was subdivided into the three phases as defined by Rank (1996). Also included are the similar phases defined for an OSSE analysis (Murphy and Share 1999) and an EGRET/TASC analysis (Dunphy et al. 1999).

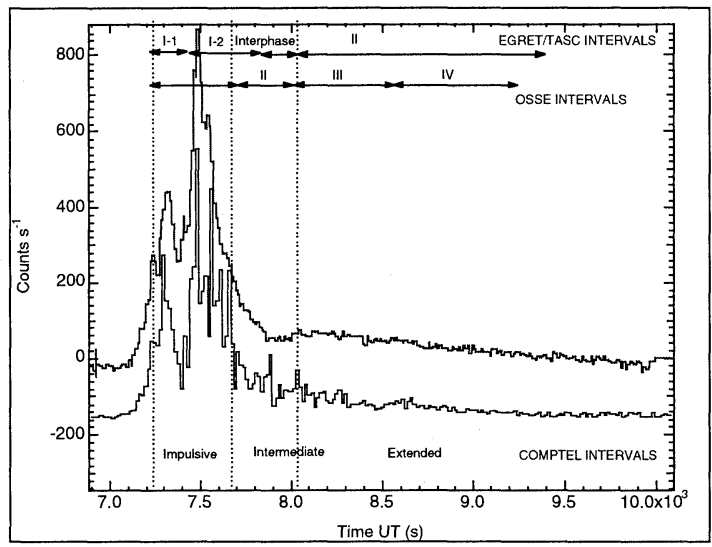

FIGURE 1. Light curves of the 11 June $1991 \mathrm{X}$-class solar flare as measured by the COMPTEL telescope (lower-blue) and burst modes (upper-green). Included are the observation intervals for COMPTEL, OSSE, and EGRET/TASC. The curves are slightly offset arbitrarily.

The COMPTEL (see Schönfelder et al. 1993 for a discussion of COMPTEL) event data (full source and 4 background sets) were binned in energy space. These spectra were deconvolved (using a new deconvolution technique, see Young et al. 2001) with the telescope response generated for the 11 June 1991 flare (based on its location within the COMPTEL FoV). Figure 2 is the photon flux spectrum for the full flare. Included in the plot is the best fit model composed of the 19 flare SMM broad line template (Share and Murphy 1995), a power law for the electron bremsstrahlung component (determined with BATSE data and PHEBUS (Trottet et al. 1993)), the 10 strongest narrow lines (based on flare modeling) and another power law to account for pion decay secondary Bremsstrahlung.

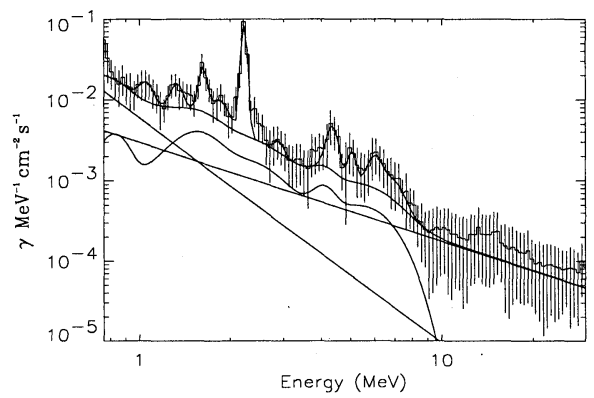

FIGURE 2. The COMPTEL flux spectrum for the entire 11 JUNE 91 flare. The 2 straight solid lines are from the bremsstrahlung model fit (steeper is primary component - see text). The solid line rolling over at $10 \mathrm{MeV}$ is the broad line model fit. The solid line just above that is the sum of the 2 bremsstrahlung components and the broad line model. The solid line closely following the data is the sum of all the models including the narrow line component. 
We have separately analyzed the data for each of the 3 phases (impulsive, intermediate, and extended) (See Figure 1) but we only discuss the intermediate phase here.

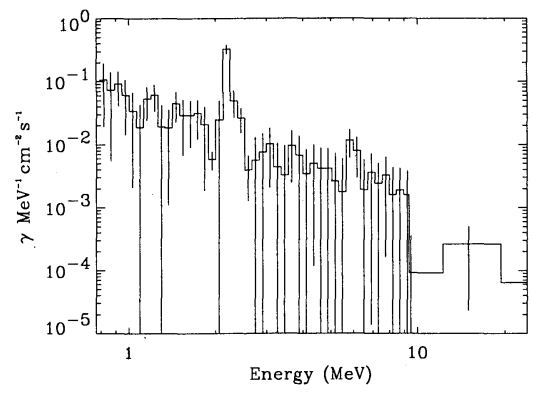

FIGURE 3. COMPTEL flux spectrum for the intermediate phase of the 11 JUNE 91 flare. (Error bars extending to the bottom of the plots are $1 \sigma$ upper limits.)

Figures 3 contain a plot of the deconvolved photon flux for the intermediate phase. The Intermediate (Rank 1996) or Interphase (Murphy and Share 1999, Dunphy et al. 1999) immediately following the peak of the impulsive phase is difficult to explain. All three analysis of this flare using COMPTEL, OSSE and EGRET data obtained a hard proton spectrum with an index around 2 using the 2.2 to $4-7 \mathrm{MeV}$ fluence ratio. This hard a spectrum would suggests the possible presence of a high energy pion component above $8 \mathrm{MeV}$ and an emission line at 5.3 due to spallation of $\mathrm{C}$ and $\mathrm{O}$. However, none of the three instruments observed such a component. Murphy and Share (1999) argued that the inconsistencies in their measurements, in the interphase (II) and IV, indicate a two-component spectrum. Though this is plausible for the extended interval (IV), we do not agree with this conclusion for the interphase (II). We contend that this interval only contains a soft spectrum. If it did contain a hard spectrum, we would expect to see significant emission at $5.3 \mathrm{MeV}$ and above $8 \mathrm{MeV}$. This is not seen in OSSE, EGRET/TASC, and not by the more sensitive instrument COMPTEL.

Determining the amount of expected charged pion component in the data with the COMPTEL data is difficult but we can estimate the expected amount of emission at $5.3 \mathrm{MeV}$. We were not able to fit a line at $5.3 \mathrm{MeV}$ in the intermediate phase spectrum but we can estimate an upper limit for the emission. An upper limit for the measured emission at $5.3 \mathrm{MeV}$ is $0.046 \mathrm{\gamma} \mathrm{cm}^{-2}$. If the parent proton spectrum was very hard with a spectra index of 2 (as indicated by 2.2-to-4.4 fluence ratio) then based on the measured 4.44 and $6.13 \mathrm{MeV}$ flux we would expect a fluence of $0.28 \pm 0.07 \mathrm{\gamma} \mathrm{cm}^{-2}$ (Ramaty et al 1996; Mandzhavidze and Ramaty 2000). On the other hand, the softer proton spectrum of 5 (indicated by the $1.6 / 6.1 \mathrm{MeV}$ fluence ratio) would produce a fluence of $0.06 \pm 0.02 \gamma \mathrm{cm}^{-2}$. So the upper limit on the $5.3 \mathrm{MeV}$ fluence is consistent with the softer proton spectrum.

We contend that the reason a hard spectrum is indicated by the 2.223-to-4.44 ratio is simply an indication of the inability of the ratio to give reliable results and its double valueness in this regime. The ratio turns up sharply because when the spectrum is soft the only neutron production channel is the $p$ and $\alpha$ on CNO process. At these lower energies, the ratio turns up because the production of neutrons continues in this channel but the production of lines from CNO quickly turns off. So the ratio sharply turns up which is not accounted for in many of the published results

Another indicator of the soft spectrum comes from the study of the time decay of the $2.223 \mathrm{MeV}$ line. The measurements from COMPTEL have been used to model 
(Rank 1996) the $2.223 \mathrm{MeV}$ emission with a decay constant of $\tau=230 \mathrm{~s}$ (long compared to the average $100 \mathrm{~s}$ ). The time constant $\tau$ can be defined as (Prince et al. (1983)),

$$
\tau=\left[\frac{1}{\tau_{H}}+\frac{1}{\tau_{H e}}+\frac{1}{\tau_{d}}\right]^{-1} ; \tau_{H}=\frac{1.4 \cdot 10^{19}}{n_{H}} s ; \tau_{H e}=\frac{8.5 \cdot 10^{14}}{r \cdot n_{H}} s
$$

where $\tau_{\mathrm{H}}$ is the capture time on hydrogen, $\tau_{\mathrm{He}}$ is the capture time on ${ }^{3} \mathrm{He}, \tau_{\mathrm{d}}$ is the neutron decay time, $n_{H}$ is the hydrogen number density, and $r$ is the ${ }^{3} \mathrm{He} / \mathrm{H}$ ratio. $\tau$ is maximum when $r=0$ (no competing radiation less capture on ${ }^{3} \mathrm{He}$ ). So if we set $\mathrm{r}=0$ and solve for $n_{H}$ when $\tau=230 \mathrm{~s}$ we find that $n_{H}=4.56 \cdot 10^{16} \mathrm{~cm}^{-3}$. This corresponds to a depth above the base of the photosphere of about $170 \mathrm{~km}$ (Fontenla et al. 1993). For an average $r$ of $5 \cdot 10^{-05}$ (Prince et al. 1983) the depth of would be about $50 \mathrm{~km}$ above the photosphere's base. These calculations suggest that the capture time of $230 \mathrm{~s}$ means neutrons are being captured higher up in the photosphere. For neutrons to be captured at a point of lower hydrogen density and higher height in the photosphere they would have to be of lower energy or traveling at a shallow angle in the atmosphere. The existence of low energy neutrons would indicate that the proton spectrum during this part of the flare was soft not hard. So given the indication of a soft spectrum in terms of unambiguous line ratios and the extremely long $2.223 \mathrm{MeV}$ decay the most plausible explanation for the proton spectrum during the interphase is that it has a very soft spectrum.

Given a very soft proton spectrum we can estimate the ${ }^{3} \mathrm{He}$ content of the photosphere. What we will use is the neutron thermalization model we have developed (Young 2001). This will allow us to calculate the distribution of thermal neutrons in the solar atmosphere and then calculate the neutron absorption rate and decay time using a model for the density distribution for the lower chromosphere and the photosphere (Fontenla et al. 1993). Previous calculations of the ${ }^{3} \mathrm{He}$ content in the photosphere have not used information about the spatial distribution of the thermal neutrons. They only used a single average depth and so a single atmosphere density to calculate the ${ }^{3} \mathrm{He} / \mathrm{H}$ ratio. We will now use our calculations of neutron transport to calculate a more realistic ratio using the spatial distribution of the thermal neutrons. Since the protons spectrum is soft, the neutron spectrum is dominated by low energy neutrons $(<10 \mathrm{MeV})$. This allows the use of the analytical neutron transport model.

Using the distribution for thermal neutrons we calculated, we obtain a ${ }^{3} \mathrm{He} / \mathrm{H}$ ratio of $8.7 \mathrm{e}-05$ with a $1 \sigma$ range of $1.96 \mathrm{e}-04$ to $1.75 \mathrm{e}-05$. Previous values that have been reported are 5e-05 (no error reported) for the 7 June 1980 flare (Chupp et al. 1981); (2.3 \pm 1.2$)$ e-05 for the 3 June 1982 flare (Hua and Lingenfelter 1987), 2.3e-05 (2 $\sigma$

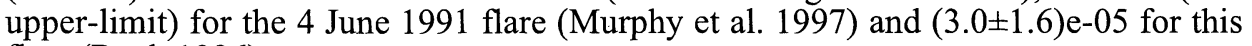
flare (Rank 1996).

The purpose of this work is to present an explanation of the puzzle presented by the spectral observations by CGRO of the intermediate phase of the 11 June 1991 solar flare. Analysis of observations by OSSE (Murphy and Share 1999), COMPTEL (Rank 1996) and EGRET (Dunphy et al. 1999) using standard spectroscopy methods indicates the presence of both a hard and a soft parent proton spectrum. We present an application of a new spectroscopy technique to the COMPTEL observations. We then show that the theoretically expected emission from a hard proton spectrum is not observed by COMPTEL. We conclude that the lack of this predicted emission and the longer than normal $2.223 \mathrm{MeV}$ emission decay time (Rank 1996) can only be due to a soft parent proton spectrum. This means that the region of 2.223/4-7 MeV fluence space is largely unexplored for soft proton spectra. The use of this ratio must be reexamined for proton spectra with indices greater than 5 or 6 . We then apply a model we developed for the transport of neutrons created from a soft proton spectrum to determine the photospheric ${ }^{3} \mathrm{He}$ abundance during this flare. We calculated a ${ }^{3} \mathrm{He} / \mathrm{H}$ 
ratio of $8.7 \mathrm{e}-05$ with a $1 \sigma$ range of $1.96 \mathrm{e}-04$ to $1.75 \mathrm{e}-05$ for this flare using this new model. This is larger than all previous values reported.

\section{ACKNOWLEDGMENTS}

This work was supported through NASA contract NAS5-26645 and NASA's Supporting Research and Technology program.

\section{REFERENCES}

1. Chupp, E. L., et al., Ap. J. Letters 244, 171 (1981).

2. Dunphy et al., Solar. Phys. 65, 2503-2504 (1999).

3. Fontenla, J. M. et al., Ap. J. 406, 319 (1993).

4. Hua, X. M. and Lingenfelter, R. E., Ap. J. 319, 555-566 (1987).

5. Kanbach, G. D., et al., Astron. \& Astrophy (Suppl. Series) 97, 349-353 (1993).

6. McConnell, M., "An Overview of Solar Flare Results from COMPTEL" in High-Energy Solar Phenomena - A New Era of Spacecraft Measurements, edited by J. M. Ryan and W. T. Vestrand, AIP Conference Proceedings 294, New York: American Institute of Physics, 1994, pp. 21-25.

7. Murphy R. J. et al., "OSSE Observations of Solar Flares" in Compton Gamma-Ray Observatory Workshop, edited by M. Friedlander et al., AIP Conference Proceedings 280, New York: American Institute of Physics, 1993, pp. 619-630.

8. Murphy R. J. et al., Ap. J. 490, 883-900 (1997).

9. Murphy R. J. and Share G. H., "Accelerated-Particle Spectral Variability in the 1991 June 11 Solar Flare" in The Fifth Compton Symposium, edited by M. McConnell and J. M. Ryan, AIP Conference Proceedings 510, New York: American Institute of Physics, 1998, p. 559.

10. Prince, T. A. et al., "The Time History of $2.22 \mathrm{MeV}$ Line Emission in solar Flares" in $18^{\text {th }}$ International Cosmic Ray Conference, Bangalore, India, 1983.

11. Ramaty, R. et al., "Solar Atmospheric Abundances from Gamma Ray Spectroscopy" in High Energy Solar Physics Workshop, edited by R. Ramaty et al., AIP Conference Proceedings 374, New York: American Institute of Physics, 1996, pp. 172-183.

12. Ramaty, R. and Mandzhavidze, N., "Particle Acceleration and Abundances from Gamma-Ray Line Spectroscopy" in High Energy Solar Physics Workshop - Anticipating HESSI, edited by R. Ramaty and N. Mandzhavidze., ASP Conference Series 206, San Francisco: Astronomical Society of the Pacific, 2000, pp. 6470.

13. Rank, "Gamma Rays and Neutrons of the Solar Flares on 11 and 15 June 1991 Measured with COMPTEL" Ph.D. Thesis, Technical University of Munich, 1996.

14. Ryan, J. M. et al., "COMPTEL Gamma Ray and Neutron Measurements of Solar Flares" in Compton GammaRay Observatory Workshop, edited by M. Friedlander et al., AIP Conference Proceedings 280, New York: American Institute of Physics, 1993, pp. 631-642.

15. Schneid, E. J. et al., "EGRET Observations of Extended High Energy Emissions from the Nuclear Line Flares of June 1991" in High-Energy Solar Phenomena - A New Era of Spacecraft Measurements, edited by J. M. Ryan and W. T. Vestrand, AIP Conference Proceedings 294, New York: American Institute of Physics, 1994, pp. 95-99.

16. Schönfelder, V. et al., Ap. J. Suppl. Series 86, 657-692 (1993).

17. Share, G.H. and Murphy, R. J., "Gamma-Ray Measurement of Energetic Heavy Ions at the Sun." in $26^{\text {th }}$ International Cosmic Ray Conference, Salt Lake City, 1999.

18. Suleiman, R. et al., "COMPTEL's Solar Flare Catalog" in High-Energy Solar Phenomena - A New Era of Spacecraft Measurements, edited by J. M. Ryan and W. T. Vestrand, AIP Conference Proceedings 294, New York: American Institute of Physics, 1994, pp. 51-54.

19. Trottet, G., et al., Astron. \& Astrophy (Suppl. Series) 97, 337-339 (1993).

20. Trottet, G., "X-Ray and Gamma-Ray Observations of Solar Flares by GRANAT" in High-Energy Solar Phenomena - A New Era of Spacecraft Measurements, edited by J. M. Ryan and W. T. Vestrand, AIP Conference Proceedings 294, New York: American Institute of Physics, 1994, pp. 3-14.

21. Young, C. A. et al., "Bayesian Multiscale Deconvolution Applied to Gamma-ray Spectroscopy" in These Proceedings, 2001.

22. Young, C. A., "Solar Flare Gamma-Ray Spectroscopy with CGRO-COMPTEL" Ph.D. Thesis, University of New Hampshire, 2001. 